Network Working Group

Request for Comments: 4536

P. Hoschka

Category: Informational

May 2006

The application/smil and application/smil+xml Media Types

Status of This Memo

This memo provides information for the Internet community. It does not specify an Internet standard of any kind. Distribution of this memo is unlimited.

Copyright Notice

Copyright (C) The Internet Society (2006).

Abstract

This document specifies the media type for versions $1.0,2.0$, and 2.1 of the Synchronized Multimedia Integration Language (SMIL 1.0, SMIL 2.0, SMIL 2.1). SMIL allows integration of a set of independent multimedia objects into a synchronized multimedia presentation.

1. Introduction

The World Wide Web Consortium (W3C) has issued specifications that define versions 1.0 [1],2.0 [2] and 2.1 [3] of the Synchronized Multimedia Integration Language (SMIL). This memo provides

information about the application/smil and application/smil+xml media types.

The definition is based on RFC 3023, which defines the use of the "application/xml" media type [4]. Before using the

"application/smil" or "application/smil+xml" media type, implementors must thus be familiar with [4] .

2. Synchronized Multimedia Integration Language

SMIL allows integrating a set of independent multimedia objects into a synchronized multimedia presentation. Using SMIL, an author can

1. describe the temporal behavior of the presentation,

2. describe the layout of the presentation on a screen,

3. associate hyperlinks with media objects, and

4. define conditional content inclusion/exclusion based on system/network properties. 


\section{Registration Information}

3.1. Registration of MIME media type application/smil

MIME media type name: application

MIME subtype name: smil

Required parameters: none

Optional parameters:

charset

Same as charset parameter considerations of application/xml in RFC 3023 .

profile

See Section 5 of this document.

Encoding considerations:

Same as encoding considerations of application/xml in RFC 3023

Security considerations: See Section 6, "Security Considerations", of this document.

Interoperability considerations:

SMIL documents contain links to other media objects. The SMIL player must be able to decode the media types of these media in order to display the whole document. To increase interoperability, SMIL has provisions for including alternate versions of a media object in a document.

Published specification: See [1], [2], and [3]

Applications which use this media type:

SMIL players and editors 


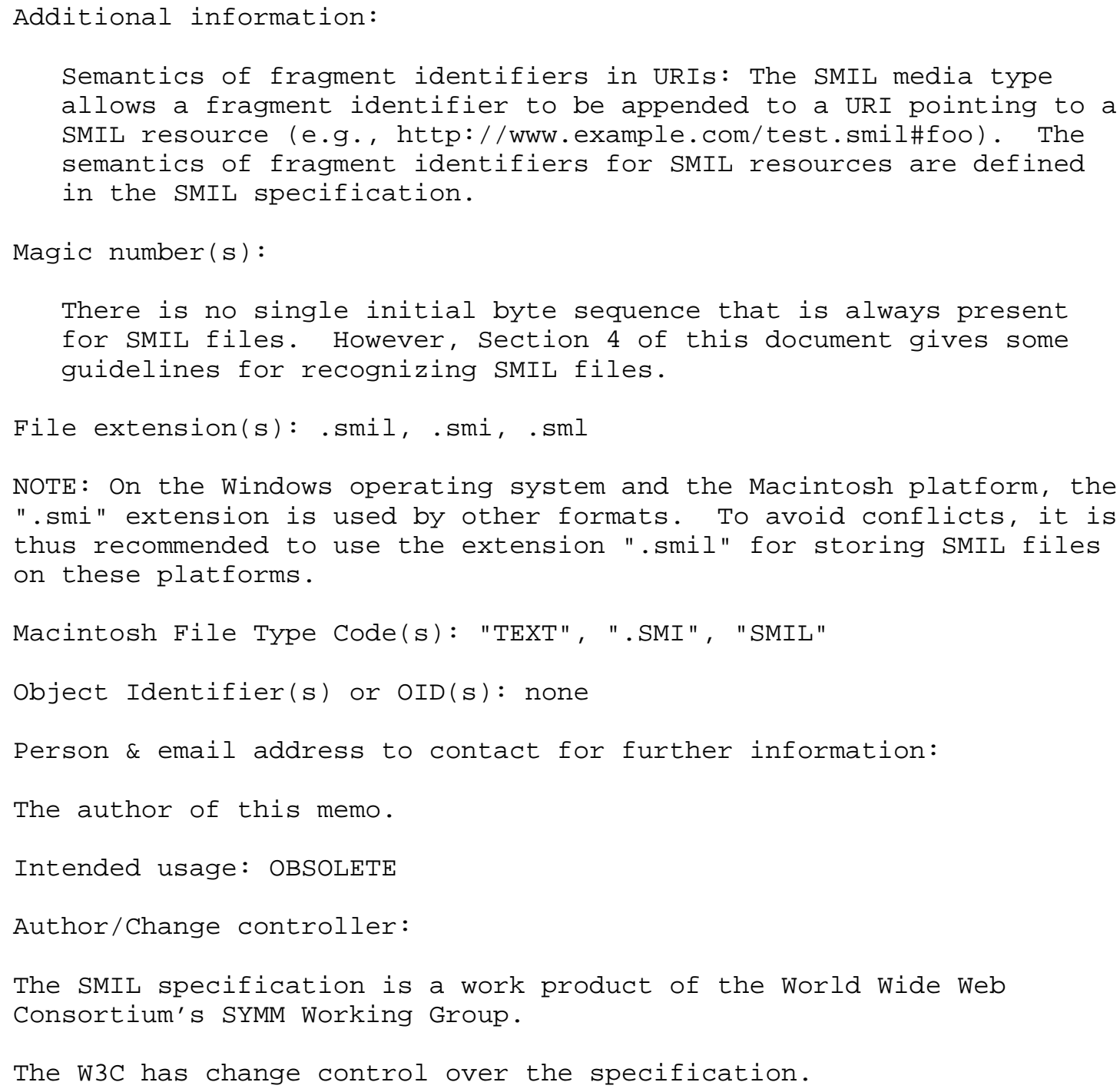




\subsection{Registration of MIME media type application/smil+xml}

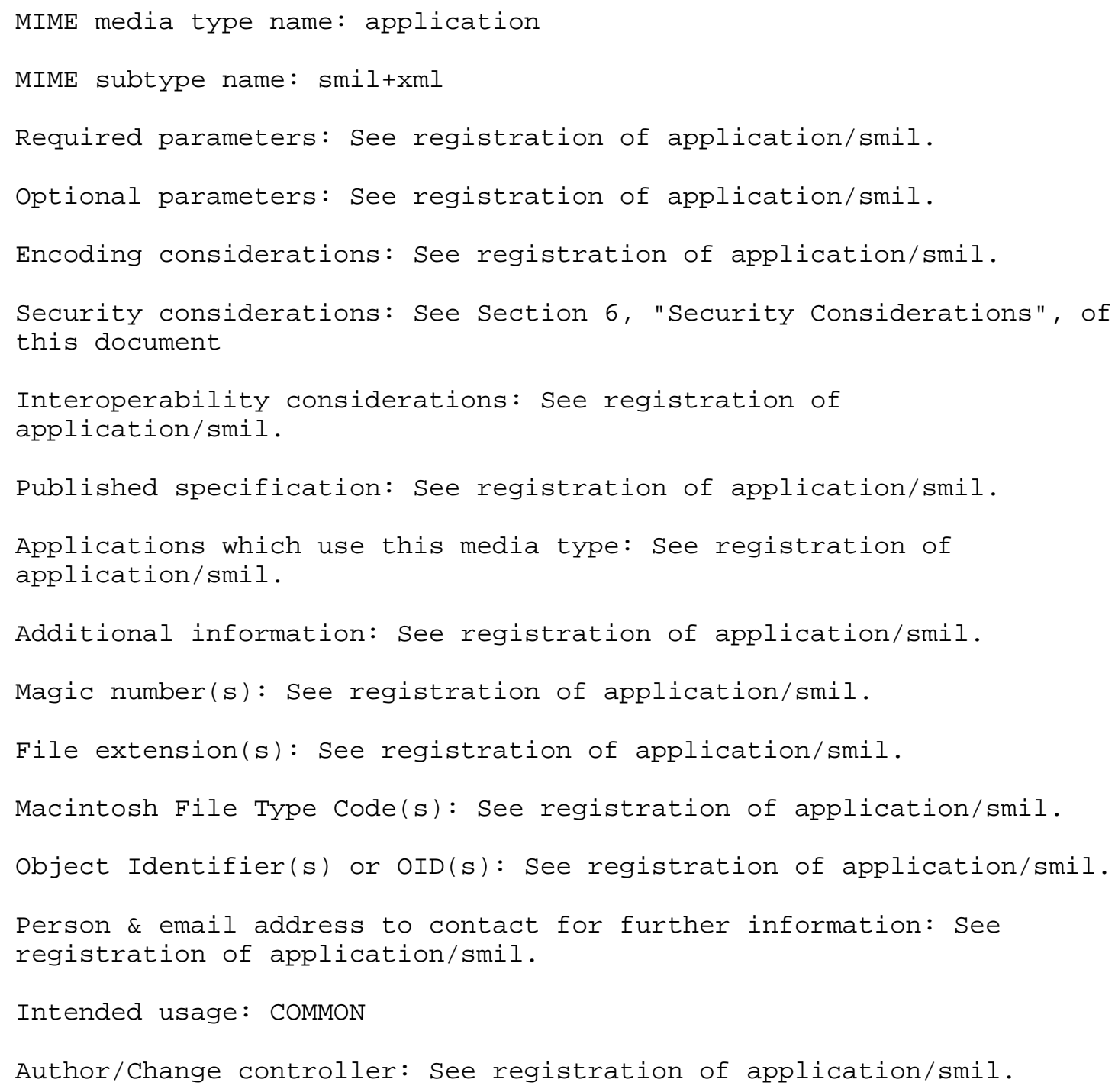




\section{Recognizing SMIL Files}

All SMIL files will have the string "<smil" near the beginning of the file. Some will also begin with an XML declaration that begins with "<? xml", though that alone does not indicate a sMIL document.

All SMIL 2.0 files must include a declaration of the SMIL 2.0 namespace. This should appear shortly after the string "<smil", and should read 'xmlns="http://www.w3.org/2001/SMIL20/Language"' .

All SMIL 2.1 files must include a declaration of a SMIL 2.1 namespace, appearing shortly after the string "<smil". The namespace string depends on the language profile. Please refer to the SMIL 2.1 specification for the definition of the relevant namespace names.

\section{The "profile" Optional Parameter}

This parameter is meant to be used in MIME media-type-based content negotiation (such as that done with the HTTP "Accept" header) to negotiate for a variety of SMIL-based languages. It is modelled after the "profile" parameter in the application/xhtml+xml MIME type registration [5] and is motivated by very similar considerations.

The parameter is intended to be used only during content negotiation. It is not expected that it be used to deliver content, or that origin web servers have any knowledge of it (though they are welcome to). It is primarily targeted for use on the network by proxies in the HTTP chain that manipulate data formats (such as transcoders).

The value of the profile attribute is a URI that can be used as a name to identify a language. Though the URI need not be resolved in order to be useful as a name, it could be a namespace, schema, or language specification.

For example, user agents supporting only SMIL Basic (see http://www.w3.org/TR/smil20/smil-basic.html) currently have no standard means to convey their inability to fully support SMIL 2.0. While SMIL 2.0 Basic user agents are required to parse the full SMIL 2.0 language, there is potentially a substantial burden in receiving and parsing document content that will not be presented to the user, since its functionality is not included in SMIL Basic.

In the future, the functionality afforded by this parameter will also be achievable by the emerging work on a protocol to transfer Composite Capability/Preferences Profiles (CC/PP) descriptions [6]. It is suggested that the "profile" parameter be used until the CC/PP protocol work has been finalized. 
An example use of this parameter as part of a HTTP GET transaction would be:

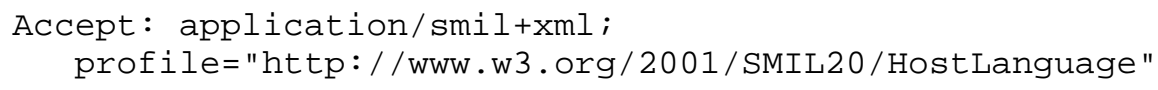

6. Security Considerations

SMIL documents contain a construct that allows "infinite loops". This is indispensable for a multimedia format. However, SMIL clients should foresee provisions such as a "stop" button that lets users interrupt such an "infinite loop".

As with HTML, SMIL documents contain links to other media (images, sounds, videos, text, etc.), and those links are typically followed automatically by software, resulting in the transfer of files without the explicit request of the user for each one. The security considerations of each linked file are those of the individual registered types.

The SMIL language contains "switch" elements. SMIL provides no mechanism that ensures that the media objects contained in a "switch" element provide equivalent information. An author knowing that one SMIL player will display one alternative of a "switch" and another will display a different part can put different information in the two parts. While there are legitimate use cases for this, it also gives rise to a security consideration: The author can fool viewers into thinking that the same information was displayed when in fact it was not.

In addition, all of the security considerations of RFC 3023 also apply to SMIL. 
7. Normative References

[1] "Synchronized Multimedia Integration Language (SMIL) 1.0 Specification", W3C Recommendation REC-smil-19980615, http://www.w3.org/TR/1998/REC-smil/, July 1998.

[2] "Synchronized Multimedia Integration Language (SMIL 2.0) [Second Edition]", W3C Recommendation, http://www.w3.org/TR/2005/REC-SMIL2-20050107/, January 2005.

[3] "Synchronized Multimedia Integration Language (SMIL 2.1)", W3C Recommendation, http://www.w3.org/TR/2005/REC-SMIL2-20051213/, December 2005 .

[4] Murata, M., St. Laurent, S., and D. Kohn, "XML Media Types", RFC 3023, January 2001 .

8. Informative References

[5] Baker, M. and P. Stark, "The 'application/xhtml+xml' Media Type", RFC 3236, January 2002 .

[6] H. Ohto, J. Hjelm, G. Klyne, M. Butler, L. Tran, F. Reynolds, C. Woodrow "Composite Capability/Preferences Profiles (CC/PP): Structure and Vocabularies 1.0", W3C Recommendation http://wWw.w3.org/TR/CCPP-struct-vocab/, January 2004.

Author's Address

Philipp Hoschka

W3C/ERCIM

2004, route des Lucioles - B.P. 93

06902 Sophia Antipolis Cedex

FRANCE

EMail: ph@w3.org 
Full Copyright statement

Copyright (C) The Internet Society (2006).

This document is subject to the rights, licenses and restrictions contained in BCP 78, and except as set forth therein, the authors retain all their rights.

This document and the information contained herein are provided on an "AS IS" basis and THE CONTRIBUTOR, THE ORGANIZATION HE/SHE REPRESENTS OR IS SPONSORED BY (IF ANY), THE INTERNET SOCIETY AND THE INTERNET ENGINEERING TASK FORCE DISCLAIM ALL WARRANTIES, EXPRESS OR IMPLIED, INCLUDING BUT NOT LIMITED TO ANY WARRANTY THAT THE USE OF THE INFORMATION HEREIN WILL NOT INFRINGE ANY RIGHTS OR ANY IMPLIED WARRANTIES OF MERCHANTABILITY OR FITNESS FOR A PARTICULAR PURPOSE.

Intellectual Property

The IETF takes no position regarding the validity or scope of any Intellectual Property Rights or other rights that might be claimed to pertain to the implementation or use of the technology described in this document or the extent to which any license under such rights might or might not be available; nor does it represent that it has made any independent effort to identify any such rights. Information on the procedures with respect to rights in RFC documents can be found in BCP 78 and BCP 79 .

Copies of IPR disclosures made to the IETF Secretariat and any assurances of licenses to be made available, or the result of an attempt made to obtain a general license or permission for the use of such proprietary rights by implementers or users of this specification can be obtained from the IETF on-line IPR repository at http://www.ietf.org/ipr.

The IETF invites any interested party to bring to its attention any copyrights, patents or patent applications, or other proprietary rights that may cover technology that may be required to implement this standard. Please address the information to the IETF at ietf-ipreietf.org.

Acknowledgement

Funding for the RFC Editor function is provided by the IETF Administrative Support Activity (IASA). 